\title{
CAN YOUNG DRIVERS LEARN TO ANTICIPATE HIDDEN HAZARDS: A DRIVING SIMULATOR STUDY
}

Siby Samuel, Tracy Zafian, Avinoam Borowsky, Matthew R. E. Romoser \& Donald L. Fisher Arbella Human Performance Laboratory, Department of Mechanical \& Industrial Engineering, University of Massachusetts, Amherst, Massachusetts, USA

Email: samuelsiby@gmail.com

\begin{abstract}
Summary: Modern technology makes possible improvements in training programs designed to develop young drivers' abilities to anticipate hazardous situations. These improvements which come from increases in the range of scenarios to which young drivers are exposed and the number of times young drivers can practice the skills they are learning. In this study, a new Flash-based, PC training program that runs on the web, Road Aware ${ }^{\circledR}$ (RA), is evaluated using a driving simulator. The program was developed by State Farm. Twenty-four young trained drivers and twenty four young untrained drivers were asked to drive various simulated hazardous scenarios while their gaze was monitored by an eye tracking system. The results show that trained drivers were more likely to anticipate hazards than their untrained peers, a difference which was present for both near transfer (scenarios that appeared in training) and far transfer scenarios. The effectiveness of RA is compared with other hazard anticipation training programs that were evaluated on a driving simulator and in the field. It appears every bit as effective in general and more effective for some scenarios. Additionally, there is evidence suggesting that, for the first time, young drivers can be trained to anticipate hazards as well as drivers who are older and more experienced.
\end{abstract}

\section{INTRODUCTION}

Young-novice drivers' hazard perception skills are poor (Pollatsek et al., 2006; Borowsky et al., 2010) and are associated with their higher likelihood of being involved in a crash as compared to more experienced drivers (e.g., Horswill and McKenna, 2004). In order to improve these skills several hazard anticipation training programs have been developed (e.g., Chapman et al., 2002; McKenna, Horswill \& Alexander, 2006; Crundall et al., 2010; Meir et al., 2010). The evidence is that such training programs do reduce crashes (Horswill and McKenna, 2004; Wells et al., 2008).

RAPT-3 (Risk Awareness and Perception Training version 3) is the only program to be evaluated both on a driving simulator (Pollatsek et al., 2006; Pradhan et al., 2006) and in the field over both the short (Pradhan et al., 2009a \& 2009b) and long (Taylor et al., 2011) term. The potential hazards embedded in RAPT-3 are ones that young drivers rarely identify (for the complete list of hazards, see Fisher, 2008, \& Pollatsek et al., 2006). RAPT-3 is effective, increasing the percentage of hazards novice drivers anticipate in the field from $38 \%$ to $62 \%$ up to a year after training, bringing the novice drivers halfway along the path to experienced drivers (82\%; Taylor et al., 2012). However, larger improvements from training would be beneficial, especially in some scenarios. For example, in some intersection scenarios no training effects have emerged after RAPT training (Pollatsek et al., 2006). Moreover, the performance of both the trained and untrained drivers in these intersection scenarios as well as a few other scenarios is near floor.

There are at least two reasons the training may not be as effective as hoped. It is well known that repetition is an important ingredient to success, where even overlearning can be of benefit (Rohrer et 
al., 2005). In RAPT-3, trainees are given only two chances to practice their hazard anticipation skills in any one scenario. Second, it is well known that concept learning occurs best when several exemplars are used in training (Hupp, 1986). In RAPT-3, only one example of each type of scenario is used.

Technological developments in recent years have allowed State Farm Mutual Automobile Insurance Company (State Farm) to develop a Flash-based, PC hazard anticipation program called Road Aware ${ }^{\circledR}$ (RA) which overcomes the above two limitations. RA trainees see the world from the driver's perspective. RA includes buttons that make it possible for trainees to scan to the sides, over their shoulders, and towards the vehicle's rear view and side mirrors. The scenarios presented in RA include those that test hazard anticipation when the hazard is obscured as well as those that test hazard anticipation when the hazard is fully visible and obviously a threat. Users see each category of scenario multiple times. In each instance, the category of scenario is presented in a slightly different manner. Thus, in the same amount of time as the RAPT-3 training, the users of RA get to practice multiple times on multiple instances of a given category of scenario, unlike in RAPT-3.

This study used a driving simulator to evaluate the effectiveness of RA for teaching young drivers to anticipate roadway hazardous situations. Specifically we examined how well young drivers who were trained in RA performed on simulator drives compared to their untrained peers. This study also evaluated the advantage of RA over RAPT-3 in terms of RA's potential to train young drivers to anticipate hazards that were not an essential part of training (far transfer). Finally, the current study compared the performance of the young drivers receiving RA training with more experienced untrained drivers.

\section{METHOD}

\section{Participants}

Forty-eight young drivers (18-22 years old) participated in the study as paid volunteers. The participants were recruited from the Amherst, MA area and the University of Massachusetts community.

\section{Equipment and Apparatus}

Driving Simulator. The advanced RTI (Realtime Technologies Inc.) driving simulator at the Arbella Insurance Human Performance Laboratory (HPL) includes a fixed base, full sized Saturn sedan. The simulated visual world is presented on three screens. The image resolution on each screen is 1024 $\times 768$.

Eye Tracker. A portable, lightweight eye-tracker developed by Applied Science Laboratories (ASL) is used to collect the eye-movement data for each participant during the virtual drives. The eye tracker, Mobile Eye XG, has a lightweight optical system consisting of an eye camera and a color scene camera mounted on a pair of safety goggles.

\section{Training Programs}

Two computer-based training programs, RA and Placebo, were used in this study. The RA training program consists of 16 interactive simulated, 3D drives, four in each of four different roadway environments: Neighborhood, Beyond Neighborhood, Downtown, and Highway. The Highway 
environment was used for practice and acclimation to the training program. One drive from each of the three remaining environments was used in this study. Each drive was 10 to 15 minutes in length. Trainees see the drives from the driver's perspective and can visually scan to their left and right sides, over their shoulders, and to the vehicle's rear and side mirrors. The vehicle's speed and lane position are controlled by the program. While driving, trainees are asked to scan the traffic environment and either click with a mouse (Figure 1, panel b) on or pan using the keyboard (panel a) towards objects and areas that present a potential hazard to drivers. Trainees receive auditory and visual feedback on whether, through their clicking and panning, they have identified important potential hazards. At the end of each drive, trainees are given the opportunity to review the most important hazards in each drive.
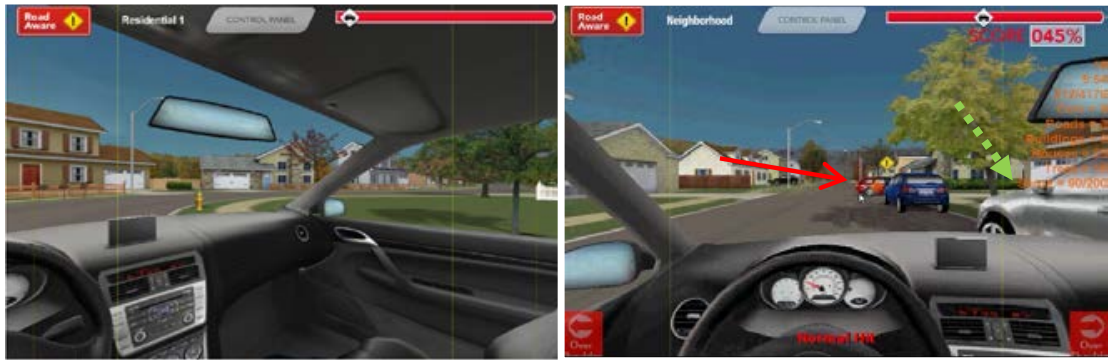

Figure 1: a) View when driver pans to the right; b) Hazard feedback: When a participant clicks on an important potential hazard (e.g., a car pulling out, red, solid arrow), the RA hazard sign appears above it (green, dotted arrow)

RA training aims to teach young drivers to identify both materialized hazards (visible or obvious hazard instigators that are clearly on a collision course with the driver and require an evasive response, e.g., a pedestrian crossing the road) and un-materialized hazards (visible or hidden hazard instigators that may materialize, e.g., a pedestrian standing on the curb who may cross the road). Each RA drive includes 3 materialized and 3 un-materialized hazardous scenarios with real-time, interactive feedback immediately after each scenario in a drive is traversed. Specifically, during each drive, at the end of the first one or two occurrences of each of the designated scenarios, the drive is paused and rewound back to the beginning of the scenario. Trainees are then given audio instructions about where they should pan or click in that scenario and why, accompanied by visually guided actions indicating where the hazardous object or area is located. Once the participant takes the correct action(s) the drive is fast forwarded to the end of the scenario and the drive continued from that point. If the participant does not take the correct action, the feedback is repeated once and then the drive continues. Finally, at the end of each drive each participant observes a Power-Point presentation where all the pre-defined scenarios and the correct actions are explained again.

The Placebo training program for this study shows participants real-world traffic scene videos and schematic views of several RAPT-3 scenarios but with no informative feedback as in RAPT-3.

\section{Simulator Evaluation Scenarios}

Near Transfer. Eighteen evaluation scenarios (9 materialized and 9 un-materialized) of one to two minutes of driving each were developed on the RTI. Each scenario was designed to replicate an RA scenario for which interactive feedback was given. The order of the eighteen simulator drives was counterbalanced across participants within each group.

Far Transfer. To evaluate how younger drivers would perform on scenarios that they did not encounter during training, we incorporated a drive with five un-materialized far transfer scenarios (e.g.: a hidden merge lane on the right) The reasons that these far transfer scenarios were hazardous 
were very similar to the reason that the scenarios were hazardous in training, but they occurred in different environments.

\section{Experimental Design}

A between-subject design was used in order to test the effectiveness of RA as a training tool. Twenty-four young drivers each, randomly selected, were assigned to the RA condition and the Placebo condition. The two groups were similar in terms of subject age, gender and driving experience. Both groups had 15 males \& 9 females. The median ages of RA and Placebo trainees were 20.3 years and 20.8 years respectively. In both groups, the median driving experience was 3.8 years. Participants in both experimental conditions completed a computer-based training program (RA or Placebo). After training (Session I) all participants drove two types of drives (Session II): (1) near transfer scenarios and (2) far transfer drive.

We focused on glances as an independent measure of evaluating drivers' ability to identify hazardous situations. Glances were defined as any sequence of fixations that were directed towards areas of the virtual world that were predetermined to be indicative of hazard identification. Identification was measured as a binary variable (1- hit, 0 - miss).

\section{Procedure}

All participants received RA or Placebo training in Session I. Over 90\% of participants returned within one or two days for Session II; the remainder returned within a week. Each participant was given a practice drive to familiarize himself or herself with the simulator dynamics following which they were fitted with the eye tracker and asked to navigate first through the various near-transfer scenarios, and then the far transfer drive.

\section{RESULTS}

The results section includes two parts. The first presents the analysis of the near transfer scenarios where only young trained and untrained drivers were included. The second part focuses on the analysis of the far transfer scenarios. Since the dependent variable is a binary one, a logit link function was used within the framework of Generalized Estimating Equations (GEE). The GEE model included a random intercept. The significance level was set at 0.05. Participants were included as a random effect. Sequential Bonferroni tests were used for all post hoc analyses.

\section{Near Transfer Scenarios Analysis}

The logistic regression model included three fixed effects: (1) Driver Group (RA and Placebo), (2) Hazard Type (Materialized vs. Un-materialized hazard), and (3) Visibility of Hazard Instigator (whether the hazard instigator was visible or obscured). Altogether the analysis included 25 hazardous elements produced by the 18 simulated scenarios (there could be more than one latent hazard in each scenario). All main effects as well as all second- and third-order interactions were included in the model. Applying a backwards elimination procedure yielded significant effects for visibility (Wald $\mathrm{X}_{1}^{2}=5.54, \mathrm{p}<.05$ ) driver group $\left(\mathrm{X}_{1}^{2}=16.12, \mathrm{p}<.01\right)$ and the interaction between visibility and driver group $\left(\mathrm{X}_{1}^{2}=16.12, \mathrm{p}<.05\right)$.

Figure 2 presents the estimated marginal means of the likelihood that participants identified the hazard in each of the four conditions, illustrating as well the interaction between Driver Group and 
Visibility of the Hazard Instigator (HI). About 47\% of the placebo-trained group anticipated hidden hazard instigators as opposed to $73 \%$ in the RA-trained group.

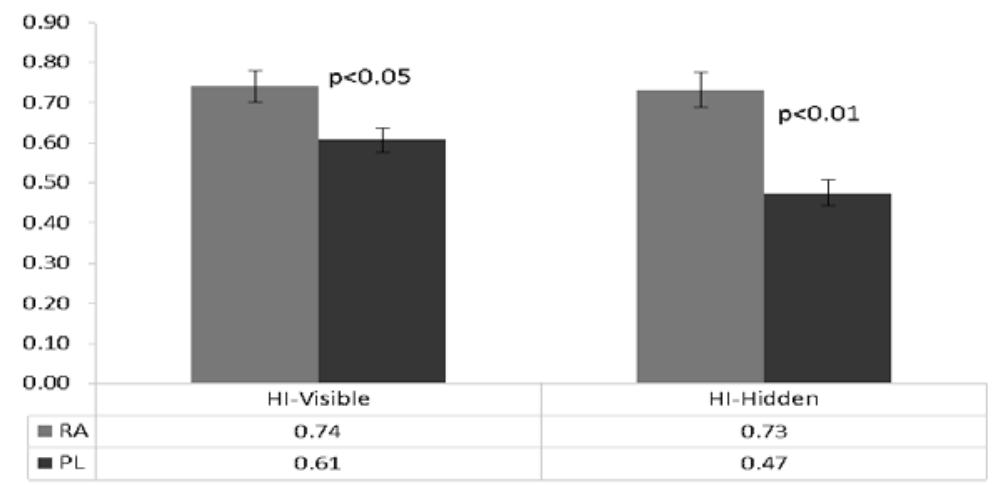

Figure 2: The Estimated likelihood of hazard identification for the interaction between Driver Group and Hazard Instigator (HI) Visibility. (HI-Visible: Hazard Instigator Visible; HI-Hidden: Hazard Instigator Hidden)

Pair-wise comparisons revealed that participants in the RA condition were significantly more likely to identify the hazard both when the hazard instigator was visible and when the instigator was hidden. The difference was largest when the hazard instigator was obscured.

\section{Far Transfer Scenarios Analysis}

The far transfer drive included the five scenarios that were not included in training and were described above. Applying a backwards elimination procedure indicated that only the Driver Group main effect was statistically significant $\left(\mathrm{X}_{1}^{2}=10.181, \mathrm{p}<.01\right)$. The data show that $\mathrm{RA}$ trainees (estimated likelihood $=0.80$, standard error $=.067$ ) identified hazards much better than Placebo trainees $(0.46, .061)$. Road Aware trained drivers perceived hazards $80 \%$ of the time as compared to $46 \%$ in the placebo condition.

\section{DISCUSSION}

The current study uses a driving simulator to evaluate the effectiveness of Road Aware ${ }^{\circledR}$, for teaching young drivers to anticipate hazardous situations. Specifically, eye movements were utilized to examine how well young drivers who were trained using RA identified hazardous situations compared with their untrained peers.

\section{Near Transfer Scenarios}

Consistent with our hypotheses, the study findings show that the performance of RA trainees was superior to that of Placebo trainees. The results of the near transfer scenarios (scenarios on the RTI on which the participants given RA were trained) show not only that RA trainees are much better at anticipating hazards in general but further indicate that when the hazard instigator was obscured (hidden), the differences between RA and placebo are larger (26 percentage points versus 13 percentage points for hidden and visible respectively). Related hazard anticipation training programs such as RAPT-2 and RAPT-3 focused only on hidden hazards. RAPT-2 is similar to RAPT-3 but utilized more diagrams and schematic views of scenarios and fewer photographs. The percentage gain in the study using RAPT-2 on the driving simulator was 25\% (Pollatsek et al., 2006). 
Of considerable importance are the results for those scenario that teach drivers to glance far enough downstream towards a curve in the road to detect the curve in time to slow. Single-vehicle, off the road crashes are a major category of young driver crashes; negotiating a curve too quickly for conditions is often the direct cause of such crashes. RA is the first hazard anticipation program of which we are aware that actually trains young drivers to glance far downstream towards bends in the road. Moreover, the training effects are large. Some $85 \%$ of the RA trained drivers in the study executed such a glance on the RTI, compared with only $41 \%$ of the Placebo trained drivers.

\section{Far Transfer Scenarios}

Analyzing the far transfer scenario reveals that not only were RA participants better than Placebo participants on scenarios presented during training but they were also better than Placebo participants in unfamiliar hazardous situations. Furthermore, for these unfamiliar scenarios RA participants demonstrated hazard anticipation skills that were almost twice as good as that of Placebo participants (80\% versus $48 \%$ ). To the best of our knowledge no training program has yet shown a comparable performance for far transfer scenarios with respect to hidden hazards that do not materialize, either on a driving simulator (e.g., 20 percentage points, Pollatsek et al., 2006) or in the field (18 percentage points, Pradhan et al., 2009).

As noted by others (e.g., Borowsky et al., submitted) younger drivers have difficulty identifying as hazardous un-controlled intersections where drivers go straight and are not required to stop. What is really rather extraordinary is that RA increases the likelihood that young drivers will anticipate hazards at such intersections (Far Transfer Scenarios 2,3 and 5 above), even when the particular scenarios which define those intersections have not been trained directly (78\% RA versus 41\% Placebo). By comparison, the effect of RAPT-2 on far transfer learning at similar simulator scenarios is very small (29\% RAPT-2 versus $23 \%$ Placebo) and the effect of RAPT-3 on far transfer learning in the field is not much larger (24\% RAPT-3 versus 14\% Placebo). We believe that a major part of the RA training effect is related to the large number and diversity of intersections that are embedded in the training program.

\section{Experienced Drivers}

Direct evidence that RA training can improve young drivers to the level of highly experienced drivers is not available. However, in a field study (Taylor et al., 2011) experienced drivers anticipated some $82 \%$ of the hazards; In the simulator study here, the young drivers trained with Road Aware ${ }^{\circledR}$ anticipated $81 \%$ of the hazards (near transfer). Both the method of evaluation (field versus simulator) and the actual scenarios trained (RAPT-3 versus RA) are different. Yet the results are encouraging.

\section{Summary}

RA demonstrates good training potential. For near transfer scenarios, the effects of training are at least as large as the effects of other training programs. Moreover, the effects extend to curves, something that has never been trained before. For far transfer scenarios, the effects appear much larger than they do when other training programs are used, especially when intersections where drivers don't have to stop are considered. Finally, the RA trained young drivers appear to be doing as well as more experienced drivers. 


\section{ACKNOWLEDGEMENT}

This research has been supported by a grant from State Farm Insurance to Donald Fisher.

\section{REFERENCES}

Borowsky, A., Shinar, D., \& Oron-Gilad, T. (2010). Age and skill differences in driving related hazard perception, Accident Analysis \& Prevention, vol. 42, pp. 1240-1249.

Chapman, P., Underwood, G. \& Roberts, K. (2002).Visual search patterns in trained and untrained novice drivers. Transportation Research Part F, 5, 157 - 167.

Crundall, D., Andrews, B., van Loon, E., Chapman, P. (2010). Commentary training improves responsiveness to hazards in a driving simulator. Accident Analysis \& Prevention, 42, $2117-$ 2124.

Fisher, D. F. (2008). Evaluation of PC-Based Novice Driver Risk Awareness. Report DOT HS 810 926. National Highway Transportation Safety Administration: Washington, DC.

Horswill, M. S., \& McKenna, F. P. (2004). Drivers' hazard perception ability: Situation awareness on the road. In S. Banbury\& S. Tremblay (Eds.), A cognitive approach to situation awareness: Theory and application (pp. 155-175). Aldershot, England: Ashgate.

Hupp, S.C. (1986). Use of multiple exemplars in object concept training: How many are sufficient? Analysis \& Intervention in Developmental Disabilities, 6, 305-317.

Meir A., Borowsky A., Oron-Gilad T., Parmet Y., \& Shinar D. (2010). “Act and Anticipate Hazard Perception Training for Young-Inexperienced Drivers” 3rd International Conference on Applied Human Factors \& Ergonomics (AHFE), Miami, 2010.

McKenna, F. P., Horswill, M. S., \& Alexander, J. L. (2006). Does anticipation training affect drivers' risk taking? Journal of Experimental Psychology: Applied, 12, 1-10.

Pollatsek, A., Narayanaan, V., Pradhan, A., \& Fisher, D. L. (2006). The use of eye movements to evaluate the effect of PC-based risk awareness training on an advanced driving simulator. Human Factors, 48, 447-464.

Pradhan, A. K., Fisher, D. L. \& Pollatsek, A. (2006). Risk Perception Training for Novice Drivers: Evaluating Duration of Effects on a Driving Simulator. Transportation Research Record, 1969, 58-64.

Pradhan, A. K., Pollatsek, A., Knodler, M. \& Fisher, D. L. (2009). Can younger drivers be trained to scan for information that will reduce their risk in roadway traffic scenarios that are hard to identify as hazardous? Ergonomics, 62, 657-673.

Rohrer, D., Taylor, K., Pashler, H., Wixted, J.T. \& Cepeda, N.J. (2005). The effect of overlearning on long-term retention. Applied Cognitive Psychology, 19, 361-374.

Taylor, T. G. G., Masserang, K.M., Divekar, G., Samuel, S., Muttart, J. W, Pollatsek, A. \& Fisher, D. L. (2012). Long-term effects of hazard anticipation training on novice drivers measured on the open road. Proceedings of Driving Assessment 2011, Lake Tahoe, Nevada. Iowa City: Public Policy Center, University of Iowa.

Wells, P., Tong, S. Sexton, B., Grayson, G. \& Jones, E. (2008). Cohort II: A Study of Learner and New Drivers Volume 1 - Main Report (Road Safety Research Report No. 81). London: TRL, DOT. 